\title{
Vibrotactile masking: A comparison of psychophysical procedures
}

\author{
RITA E. SNYDER \\ Indiana University, Bloomington, Indiana 47401
}

\begin{abstract}
The amounts of ipsilateral and contralateral masking obtained with a modified psychophysical procedure were compared for vibratory stimuli presented to different body loci. Results of a combined forced-choice localization task and a forced-choice detection task were similar to the results obtained in previous investigations which employed method of limits and two-alternative, temporal forced-choice procedures, respectively. Ipsilateral maskers produced similar amounts of masking in both the detection and localization tasks for fingertip and arm test sites. Contralateral maskers resulted in considerably more masking in the localization task than in the detection task for both fingertip and arm test sites. When large longitudinal distances were introduced between the test stimulus and masker, little masking was evident in either the localization or detection task. It was concluded that the differences in the amount of ipsilateral and contralateral masking obtained with different psychophysical procedures reflect different effects of a masker on the detectable attributes of a test stimulus. Implications of these results for the study of multiple tactile perception were discussed.
\end{abstract}

The phenomenon of tactile masking has been the subject of a number of experimental studies. The effects of such variables as the intensity of the masking stimulus, the temporal relationships between the masking and test stimuli, and the spatial relationships between the masking and test stimuli have received particular attention (Abramsky, Carmon, \& Benton, 1971; Gescheider, Herman, \& Phillips, 1970; Gilson, 1969; Halliday \& Mingay, 1961; Schmidt, 1961; Sherrick, 1964; Uttal \& Smith, 1968). The effect of spatial manipulations is the primary concern of the present study.

In 1970, Gescheider et al. reported that, similar to Sherrick's (1964) early findings, a test stimulus is masked more readily by a masking stimulus presented to the same side of the body (ipsilateral masking) than by a masking stimulus presented to the opposite side of the body (contralateral masking). The choice of psychophysical procedure was particularly important in determining the relative amounts of ipsilateral and contralateral masking that Gescheider et al. obtained. They compared the results of a signal tracking procedure, similar to that employed by Sherrick (1964), to the results obtained from a forced-choice procedure. In the forced-choice procedure, observers compared a stimulus presentation of the masker alone with one in which both

This research was supported by Grant NS-09783 from the National Institutes of Health, U.S. Department of Health, Education, and Welfare. I would like to thank James Craig for his helpful comments and advice on all aspects of this research. The author's present address: Department of Psychology, Denison University, Granville, Ohio 43023. masker and test stimulus appeared. Gescheider et al. found that ipsilateral masking was identical for both procedures. With a contralateral masker, the amount of masking was much less with the forced-choice method than with the tracking procedure. Such a finding has a direct bearing on the conclusions which may be drawn from masking studies concerning the way in which spatially distinct stimuli are processed. Masking obtained with the more classical tracking procedure was taken by Gescheider et al. to reflect the ability to detect the test stimulus itself, while masking obtained with a forced-choice procedure was taken to reflect the ability to detect a change in the overall pattern of stimulation.

Gilson (1969) reported no difference between ipsilateral and contralateral masking when stimuli are presented to a body site such as the thigh using the method of limits procedure. This finding led Gilson to suggest that there are some differences between the ways that mechanical stimuli presented to the fingers and to other body loci are processed.

The comparison between the amounts of ipsilateral and contralateral masking obtained with different psychophysical procedures was extended to body loci in a study by Snyder (1973). First, the results obtained by Gescheider et al. were replicated in a direct comparison of the methods of limits and twoalternative, temporal forced-choice (2ATFC) procedures, using vibrotactile stimuli presented to the fingertips. Second, a comparison of the amounts of masking obtained at the volar forearm with these two procedures showed results similar to those obtained at fingertips sites: while little difference was obtained between ipsilateral and contralateral 
masking when the method of limits was employed (in agreement with Gilson, 1969), a marked ipsilateralcontralateral masking difference was obtained from the $2 \mathrm{ATFC}$ procedure.

Gilson (1974) has attempted to account for the differences in results obtained with different psychophysical procedures by extending the analysis proposed by Gescheider et al. He suggests that the different psychophysical procedures employed in masking studies involve performing essentially two different tasks. With the method of limits procedure, the observer must identify the presence of the test stimulus at a particular site. An intense masker displaces the sensation produced by the test stimulus towards the locus of the masker. This observation was first reported by von Frey and Pauli in 1913 (cited by Schmidt, 1961) and later by Békésy (1960). Assuming that the proximity of these stimuli is directly related to the degree of displacement, more displacement occurs with an ipsilateral masker than with a contralateral masker. Thus, in order to perform the localization-related task demanded by the method of limits, the test stimulus must be more intense in the presence of an ipsilateral masker to overcome the displacement, resulting in a greater difference between thresholds measured in the absence of and in the presence of a masking stimulus.

With the forced-choice procedure, Gilson (1974) suggests that such displacements are of little consequence, since the task demanded by this procedure involves recognition of a difference between the overall pattern produced by the masker alone and that produced by the combined presence of the masker and test stimulus. In the latter case, the test stimulus only needs to be intense enough to produce a discriminable change in the overall intensity of stimulation. The change in overall intensity occurs at test stimulus intensities below those needed for localization, and hence less masking is measured with this procedure.

It is clear that the procedure employed in a masking paradigm may be an important determinant of the amount of masking that is obtained as well as the interactions among different body loci that are exhibited in these studies. If a masking paradigm is to be useful in examining multiple stimulus perception, it is first necessary to stipulate the types of perceptual phenomena that may be involved in this type of investigation. The conflicting results obtained in previous studies may, in part, be explained by an analysis of the perceptual task that is being performed by the observer: a localization task is involved when a method of limits procedure is employed, and a simple detection task is involved when a forced-choice procedure is employed. The present investigation was designed to compare directly the amount of masking obtained at different body loci when localization and detection tasks are performed in the presence of a masker. The following predictions were made. First, for test stimuli and maskers placed on the fingertips and on a body locus such as the arm, masked thresholds for the localization and detection tasks should be similar when an ipsilateral masker is employed, but the masked threshold for the localization task should be higher than that for the detection task when a contralateral masker is employed (cf. Gilson, 1969; Sherrick, 1964, for a method of limits procedure; Gescheider et al., 1970; Snyder, 1973, for a forced-choice procedure). Second, when large longitudinal separations are introduced between the test stimulus and masker, there should be little difference between masked thresholds for the localization and detection tasks when either an ipsilateral or contralateral masker is employed (cf. Gilson, 1969, for a method of limits procedure; Snyder, 1973, for a forced-choice procedure).

\section{METHOD}

\section{Observers}

The observers were two women and one man for threshold measurements on the fingertip and one woman and two men for threshold measurements on the arm. The latter three observers had participated previously in similar experiments. All observers were paid an hourly rate and were given several hours of practice before data collection was begun.

\section{Apparatus}

The test and masking stimuli consisted of a $160-\mathrm{Hz}$ sinusoidal vibration, $200 \mathrm{msec}$ in duration, with a rise-fall time of $10 \mathrm{msec}$. The test and masking stimuli were in phase and had simultaneous onset times.

Sinusoidal signals were generated by a Hewlett-Packard 3300A function generator and led to two Grason-Stadler 1287 electronic switches. The electronic switches were controlled by GrasonStadler Series 1200 programming modules. The output of each electronic switch was amplified by a Krohn-Hite DCA-10 wide-band amplifier and attenuated by a Hewlett-Packard 350D attenuator. The output of each attenuator was fed through a matching transformer to a Goodmans V -47 vibrator, which had been calibrated for $160-\mathrm{Hz}$ stimuli with a MB Electronics vibration meter, Model M7.

Each vibrator was fitted with a circular plastic contractor, $6 \mathrm{~mm}$ in diameter, which protruded through a 9 -mm-diam hole in a fixed metal surround. The vibrators were placed on balance pans and a constant $20 \mathrm{~g}$ force was maintained between the contactors and the skin. The observer wore earphones at all times through which white noise was fed to mask sounds produced by the vibrators and other noises associated with the operation of the apparatus. The observer was also provided with a subject station containing lights which indicated the various intervals of each observation period.

\section{Loci}

For threshold measurements at the fingertip loci, two test sites and two masker sites were employed. The iwo test sites were the index and little fingertip of the right hand (Points 1 and 2 , Figure 1), and the ipsilateral and contralateral masker sites were the right and left thenar eminence, respectively (Point 3, Figure 1). For threshold measurements at body loci, two test sites and four 
masker sites were employed. The test sites were on the ventral surface of the upper right arm (Points 6 and 7, Figure 1). Ipsilateral and contralateral masker sites were centered on the right and left volar forearm, respectively (Point 4, Figure 1) and the right and left ventral thigh surfaces, respectively (Point 8 , Figure 1).

\section{Procedure}

A modification of the block-up-and-down 2ATFC procedure described by Campbell (1963) was employed for all threshold measurements. For each observation trial, the masker occurred in both intervals, and the test stimulus occurred in only one interval and at only one of the two test sites. Lights were used to signal the various intervals in the observation to the observer. A 1 -sec ready light was followed by two 1.5 -sec observationinterval lights, a 3-sec response-period light, and a 0.75 -sec feedback light. The observer verbally indicated both the interval and the locus of the test stimulus. The intensity required for the correct identification of the interval and locus of the test stimulus was determined in separate blocks of 20 trials. For the detection threshold measurements, the intensity of the test stimulus changed only on the basis of the observers' responses about the interval containing the test stimulus. Similarly, for the localization threshold measurements, the intensity changed only on the basis of responses concerning locus.

Before beginning the experiment, unmasked thresholds for each masker site were obtained using the $2 \mathrm{ATFC}$ detection procedure. The median of 720 observations for each observer provided the referent for each experimental masker intensity. For the fingertip maskers, intensities of 18 and $36 \mathrm{~dB}$ SL were employed, and for the body site maskers, the intensity was set at $15 \mathrm{~dB} \mathrm{SL}$.

In each experimental session, unmasked and masked thresholds were measured at either the fingertip or arm test sites under both the detection and localization procedures. Data collection was continued until a total of 400 observations was obtained from each observer for each masker locus and intensity, under both the detection and localization procedures.

\section{RESULTS}

Figure 2 shows the median change (in decibels) for the three observers in thresholds at the fingertips resulting from the presence of the masker for both detection and localization as a function of masker intensity. These median threshold changes are taken as the measure of masking for the present study. All masked thresholds are shown relative to the unmasked threshold for detection. Little difference is seen between the detection and localization tasks when thresholds are measured in the absence of a masker or when an ipsilateral masker is employed. However, with a contralateral masker, there is a small but consistent difference between the amount of masking for localization and detection. In addition, the contralateral masking difference between localization and detection appears to increase with masker intensity, while the ipsilateral masking difference between localization and detection thresholds remains consistently small.

Figure 3 shows the median change (in decibels) for the three observers in detection and in localization thresholds at the upper arm locus as a function of masker presence and locus. All changes are measured from the unmasked threshold for detection.

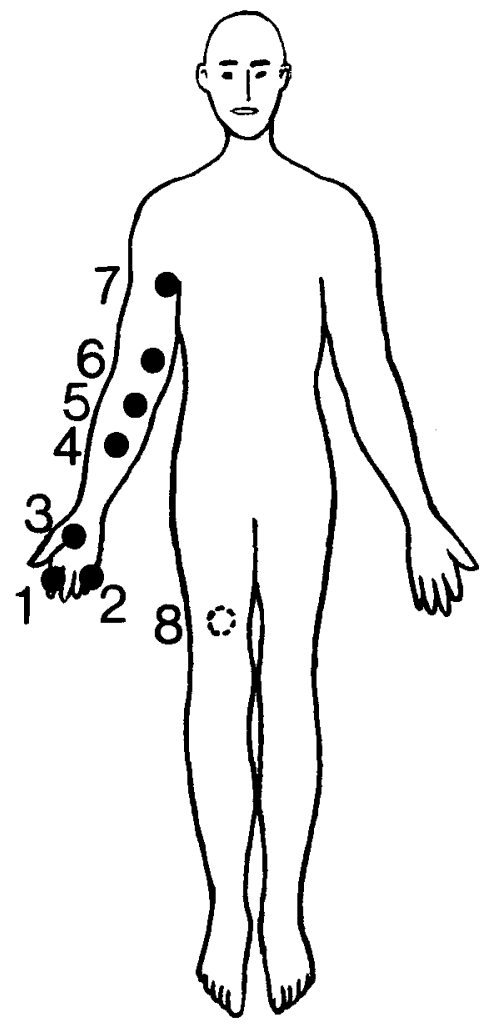

Figure 1. Locations of test stimulus and masker sites used in the present investigation.

With no masker, the threshold for localization is approximately $3 \mathrm{~dB}$ higher than the threshold for detection, resulting in different baselines from which masking effects are obtained. In general, the arm masker produces more masking than the thigh masker. For the localization task, little difference is

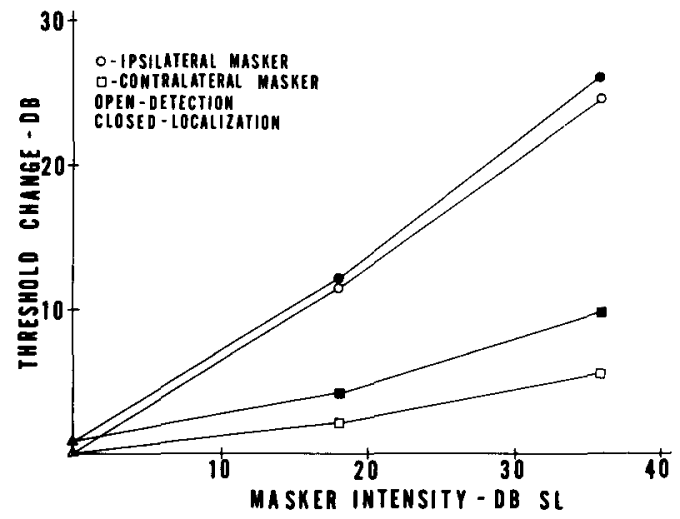

Figure 2. Localization and detection at the fingertips. Comparison of threshold changes for the localization and detection tasks as a function of ipsilateral and contralateral masker intensity. Zero dB SL is taken as the detection threshold when no masker is employed. The data presented are medians based on the results from three observers. $(\Delta=$ absolute threshold. $)$ 


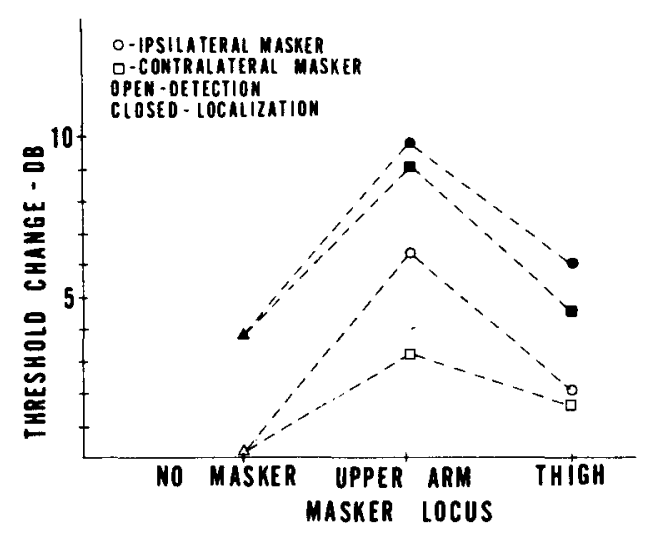

Figure 3. Localization and detection at the upper arm. Comparison of threshold changes for the localization and detection tasks as a function of masker locus. Zero dB SL is taken as the detection threshold when no masker is employed. The data presented are medians based on results from the three observers ( $\Delta=$ absolute threshold.)

seen between the threshold changes produced by an ipsilateral and a contralateral masker, regardless of the longitudinal separation between the test stimulus and the masker. For the detection task, the ipsilateral arm masker produces considerably more masking than the contralateral arm masker; however, when a large longitudinal separation is introduced, both ipsilateral and contralateral masker placement produce the same small threshold change.

Figure 4 shows the data of Figure 3 replotted to illustrate more clearly the difference (in decibels) between the masked thresholds obtained from the localization and detection procedures. The difference between the procedures is consistent when an ipsilateral masker is employed on the arm and thigh, and when no masker is employed. When a contralateral masker is presented, the localization threshold obtained with the arm maskers is increased more

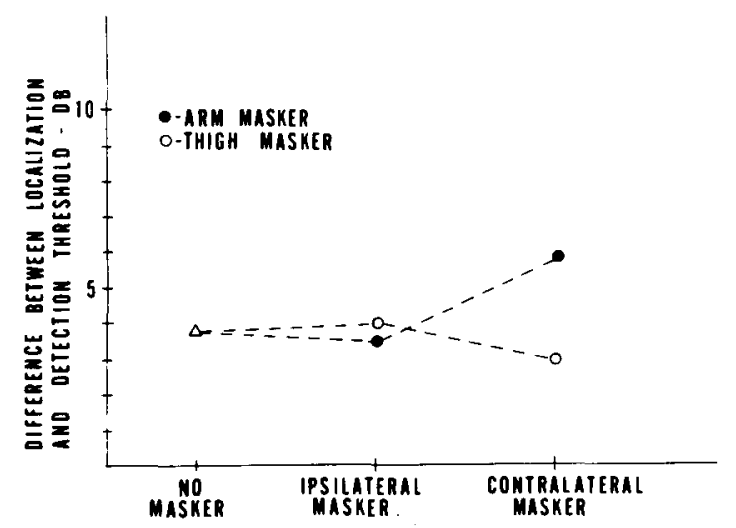

Figure 4. Difference between localization and detection thresholds at the upper arm for ipsilateral and contralateral maskers at the upper arm and the thigh. The data presented are medians based on results from the three observers. ( $\Delta=$ absolute (hreshold.) than the detection threshold; no relative change between thresholds for these two tasks is seen when the contralateral masker is placed on the thigh.

Further data analyses showed that these results were not influenced by response bias for either the interval or locus choices or by criterion changes across the different conditions. Thus, it can be assumed that the threshold differences presented in the figures accurately reflect changes in sensitivity under the task conditions employed in this study.

\section{DISCUSSION}

There appears to be a clear parallel between the results of previous studies which employed forcedchoice and classical psychophysical procedures and the present results from the detection and localization procedures, respectively. Little difference between detection and localization was seen when ipsilateral maskers were used for test sites on the fingertips and on the body. Such a result is consistent with the comparison of the amounts of masking obtained with forced-choice and classical psychophysical procedures reported by Gescheider et al. (1970) and Snyder (1973). Contralateral maskers resulted in considerably more masking for the localization task than for the detection task for both fingertip and body test sites. Such a difference in masking is consistent with previous findings that forcedchoice procedures resulted in less contralateral masking than classical psychophysical procedures in studies which employed both fingertip and body test sites (Gescheider et al., 1970; Gilson, 1969; Sherrick, 1964; Snyder, 1973). When large longitudinal distances were introduced between the test stimulus and masker, little masking was evident for either a localization or a detection task, similar to results obtained with methods of limits and forcedchoice procedures (Gilson, 1969; Snyder, 1973).

Gilson (1974) suggested that the different psychophysical procedures might be applied to investigate different types of problems. He recommended that studies which focus on examining neurological relationships among various sites would be better served by the forced-choice method. The present investigation employed this method to examine both detection and localization of stimulation at a test site and found that quite different types of relationships between stimulation at two sites result as a function of the task. Detection tasks showed similarly small amounts of masking when contralateral maskers were employed at body sites. Since Gilson has suggested that this method may be "appropriate for assessing neurological 'funneling' interactions"' (p. 54), it must be concluded that either neurological relationships are similarly weak when combinations of sites other than homolateral ipsilateral ones on the body are employed or that a detection 
procedure will not prove to be sensitive enough for assessing these relationships.

On the other hand, when a forced-choice localization procedure was employed, clear differences in the amounts of contralateral masking produced by different test-site/masker combinations were obtained. The greatest amount of contralateral masking was obtained when the masker was placed homolateral to the test site; in fact, at the arm site, a contralateral masker produced nearly as much masking an an ipsilateral masker. When large longitudinal distances were introduced between test and masking stimuli placed on the body, the amounts of ipsilateral and contralateral masking produced remained comparable, but showed a considerable decrease from the former condition. When test and masking stimuli were placed on the fingertips, a clear difference between the effects of an ipsilateral and contralateral masker were observed. These results support Gilson's (1969) suggestion that there may be differences between the ways that stimuli presented to the fingertips and to other body sites are processed, as well as point to clear differences in the amounts of masking apparent for different combinations of body sites. Since such differences are greater for a localization task than a detection task, it is suggested that investigations of neurological relationships could profit more by focusing on this aspect of tactile stimulus processing.

It has also been suggested (Gilson, 1974) that masking paradigms may be useful in specifying conditions under which stimulus identification may be affected in tactile arrays which use a large number of stimulators (e.g., Bach-y-Rita, 1972; Bliss, Katcher, Rogers, \& Shepard, 1970). Since pattern recognition depends in large part on localizing the stimulated elements, the results of the localization task may suggest useful approaches for attempts to increase discriminability in such arrays. For fingertip stimulators, considerably less interference with detection of pattern elements should result from using contralateral, rather than ipsilateral, fingers to increase the stimulator area. By contrast, stimulators applied to body surfaces may achieve similar benefits by spreading the arrays longitudinally rather than laterally. It is not clear, however, that studies such as the present one will contribute more than the most general types of approaches for the development of complex arrays. The amounts of masking evident from both the localization and detection tasks used in combination in the present study were less than the amounts obtained in previous studies which employed only one type of task. In the present study, observers made judgments about two stimulus features (presence and locus), and the type of perceptual strategy employed in making this more complex judgment may differ significantly from that used in a simple forced-choice or method of limits task. Since task complexity is increased even further when large patterns are employed, generalizing from more simple masking studies to pattern perception studies may be difficult. A more appropriate generalization from the results of the present investigation may be to recognize the importance of examining masking directly for the specific type of task that an observer must perform in identifying patterns presented through complex tactile arrays.

\section{REFERENCES}

Abramsky, O., Carmon, A., \& Benton, A. L. Masking of and by tactile pressure stimuli. Perception \& Psychophysics, 1971, 10, 353-355.

BACH-y-RITA, P. Brain mechanisms in sensory substitution. New York: Academic Press, 1972.

BÉKÉsY, G. voN. Experiments in hearing. New York: McGrawHill, 1960.

Bliss, J. C., Katcher, M. G., Rogers, C. H., \& Shepard, R. P. Optical-to-tactile image conversion for the blind. IEEE Transactions on Man-Machine Systems, 1970, MMS-11, 58-65.

CAMPBELL, R. A. Detection of a noise signal of varying duration. Journal of the Acoustical Society of America, 1963, 35, $1732-1737$.

Gescheider. G. A., Herman, D. D., \& Phillips, J. N. Criterion shifts in the measurement of tactile masking. Perception \& Psychophysics, 1970, 8, 433-436.

Gilson. R. D. Vibrotactile masking: Some spatial and temporal aspects. Perception \& Psychophysics, 1969, 5, 176-180.

GiLson, R. D. Vibratory masking. In F. A. Geldard (Ed.), Cutaneous communication systems and devices. Austin, Tex: Psychonomic Society, 1974. Pp. 53-56.

Halliday, A. M., \& Mingay, R. Retroactive raising of a sensory threshold by a contralateral stimulus. Quarterly Journal of Experimental Psychology, 1961, 13, 1-11.

SCHMIDT. E. Temporal aspects of cutaneous interaction with twopoint electrical stimulation. Journal of Experimental Psychology, $1961,61,400-409$

SHERRICK, C. E. Effects of double simultaneous stimulation of the skin. American Journal of Psychology, 1964, 77, 42-53.

SNYDER, R. Vibrotactile masking: $A$ comparison of psychophysical procedures. Unpublished doctoral dissertation, Indiana University, 1973.

UtTal, W. R., \& Smith, P. Further studies on the psychophysics of irregular nerve action patential patterns. Perception \& Psychophysics, 1968, 3, 341-345.

(Received for publication July 2, 1976; revision accepted July $8,1977$. ) 